\title{
Slat Cove Unsteadiness: Effect of 3D Flow Structures
}

\author{
Meelan M. Choudhari* and Mehdi R. Khorrami* \\ NASA Langley Research Center, Hampton, VA, 23681-2199
}

\begin{abstract}
Our previous studies have indicated that 2D, time accurate computations based on a pseudo-laminar zonal model of the slat cove region (within the framework of the Reynolds-Averaged Navier-Stokes equations) are inadequate for predicting the full unsteady dynamics of the slat cove flow field. Even though such computations could capture the large-scale, unsteady vorticity structures in the slat cove region without requiring any external forcing, the simulated vortices were excessively strong and the recirculation zone was unduly energetic in comparison with the PIV measurements for a generic high-lift configuration. To resolve this discrepancy and to help enable physics based predictions of slat aeroacoustics, the present paper is focused on 3D simulations of the slat cove flow over a computational domain of limited spanwise extent. Maintaining the pseudo-laminar approach, current results indicate that accounting for the threedimensionality of flow fluctuations leads to considerable improvement in the accuracy of the unsteady, nearfield solution. Analysis of simulation data points to the likely significance of turbulent fluctuations near the reattachment region toward the generation of broadband slat noise. The computed acoustic characteristics (in terms of the frequency spectrum and spatial distribution) within short distances from the slat resemble the previously reported, subscale measurements of slat noise.
\end{abstract}

\section{Introduction}

With the advent of quieter, ultra-high-bypass-ratio engines, flow unsteadiness in the vicinity of various airframe components has emerged as an important contributor to the noise signature of subsonic commercial transports during their approach for landing. The major sources of airframe noise include high-lift devices (i.e., leading-edge slat and trailing-edge flaps) and the aircraft undercarriage. The ranking of these sources is configuration dependent; typically, noise due to high-lift system dominates for the medium sized aircraft, whereas landing gear noise could be more important for the larger twin-engine transports. Model scale tests ${ }^{1-6}$ have identified the leading-edge slat as a prominent source of airframe noise during approach conditions. These findings are corroborated by the flyover noise measurements for the Airbus $\mathrm{A} 340^{7}$.

Slat noise generation represents a complex aeroacoustic problem (Fig. 1), resulting in both broadband noise and one or more narrower peaks that are suggestive of aerodynamic and/or aeroacoustic resonances. Previous measurements indicate that the broadband spectrum has a maximum near St $\approx 1-3$ (where the Strouhal number St $\equiv$ $\mathrm{fc}_{\mathrm{s}} / \mathrm{U}_{\infty}$ is based on the free-stream velocity $\mathrm{U}_{\infty}$ and slat chord $\mathrm{c}_{\mathrm{s}}$ ), and exhibits peak directivity in the aft quadrant at the corresponding frequency. ${ }^{8}$ The overall sound pressure level (SPL) for slat noise sources has been noted to scale with $\mathrm{M}^{4.5}[8], \mathrm{M}^{5}[9,10]$, or with $\mathrm{M}^{5}$ overall but with $\mathrm{M}^{4 . x}$ in the range of mid to high frequencies [6], where $\mathrm{M}$ denotes the free-stream/flight Mach number. However, as discussed in Refs. 8 and 10, the physical mechanisms underlying the observed spectral characteristics of slat noise are not clear at this time. This paper presents computational simulations of the slat cove flow, with the eventual goal of enabling physics based predictions of the broadband noise source(s).

Prediction techniques and noise reduction concepts for airframe noise sources have been largely empirical in nature, mainly due to the complexity of the unsteady flow around high-lift devices and landing gears. However, given the aggressive noise reduction targets set forth by NASA and the European vision for 2020, and the fact that meeting those goals may well require radical departures from the airframe configurations included in the existing database $^{11}$, the need for physics based approach to airframe noise prediction has become increasingly apparent in recent years. An essential ingredient to developing the physics based approach involves synergistic combinations of experiments and numerical simulations for unit configurations. Recent studies of this type have provided a number of useful physical insights into the noise source mechanisms that are responsible for the dominant features of the measured slat- and flap-noise spectra. Specifically, the high-frequency spectral hump in the slat noise measurements at $\mathrm{NASA}^{2,5}$ has been attributed to vortex shedding from a finite thickness slat trailing edge ${ }^{12-13}$, whereas the

\footnotetext{
* Aerospace Technologist, Computational Aerosciences Branch, Associate Fellow AIAA
} 
broadband component of slat noise at lower frequencies has been linked with the interaction between unsteady vorticity structures in the slat cove region and the adjacent features of the high-lift geometry (viz., the slat trailing edge and the gap region between the slat and the main element) ${ }^{14-16}$. See, also, [17] and [18] for additional references pertaining to the recent work on slat noise. Even though the earlier computations related to slat noise had some serious deficiencies as described in [16] and [19], those studies helped clarify the cause-effect relationships between the near-field flow structures and the far-field noise. The knowledge of such causal relationships is crucial to developing practical noise reduction concepts that are both robust and aerodynamically efficient. Time accurate simulations of high-lift configurations are, of course, essential for aeroacoustic predictions. However, such simulations can also play a vital role in advancing the state-of-the-art in purely aerodynamic predictions of high-lift performance. ${ }^{20,21}$

To serve as a basis for the quantitative predictions of broadband slat noise, the time accurate predictions of the slat cove flow must be carefully validated against an appropriate set of measured data. Towards that goal, a joint study involving particle image velocimetry (PIV) measurements and 2D unsteady computations for a generic, unswept three-element high-lift configuration was described in Refs. [22] and [19], respectively. The objective behind that work was to shed additional light on the unresolved questions pertaining to the development of unsteady flow structures within the slat cove region (including the recirculation zone of the cove) and the interaction between these structures and the adjacent solid surfaces. Specifically, the $2 \mathrm{D}$ computations ${ }^{19}$ sought to evaluate the accuracy of the previously employed zonal turbulence model, ${ }^{15}$ in which the turbulence production term was switched off within the slat cove region. Consistent with the assessment in [16], the results of these computations ${ }^{19}$ indicated excessively energetic vorticity structures in comparison with the PIV measurements, ${ }^{23}$ regardless of the main element angle-of-attack within the range of 4 to 8 degrees. No major improvements were noted in an additional set of $2 \mathrm{D}$ computations involving modifications to the zonal model, such as either a partial restoration of turbulence production within a thin region adjacent to the lower slat surface or a 2D implementation of a RANS-LES hybrid model. In an attempt to rectify the differences between measurements and the CFD data, the present paper extends those computations to include the effects of 3D fluctuations. The 3D computations are also essential to allow accurate predictions of the far-field acoustic intensity. To render such 3D computations feasible with the available computational resources, the unsteady slat-cove flow is assumed to be statistically homogeneous over the length scale of the model span, i.e., the effects of finite span length for a free-flight configuration or 3D effects due to the installation of a full-span model within a closed section wind tunnel ${ }^{24}$ are neglected in this study.

Large eddy simulations of the slat cove region for a proprietary high-lift configuration were presented earlier in Ref. [25], while detached eddy simulations for a high-lift configuration used during the EUROPIV2 program have been presented in Ref. [26]. The latter work (which was published after the completion of the present effort) demonstrates encouraging comparison between the computed and measured mean flows. In addition to presenting an independent comparison of this type for a different high-lift configuration, the present paper examines the details of the unsteady flow characteristics of the slat cove region and their likely implications for noise generation.

\section{Flow Configuration and Simulation Methodology}

The combined geometric and physical complexity of a high-lift flow configuration poses major challenges for both experiments and numerical computations. Difficulties in performing meaningful high-lift experiments include a sensitivity to flow Reynolds number (i.e., scaling effects), large-scale 3D effects even when the geometry is nominally 2D, a lack of convenient and non-intrusive access to the flow features to be measured, and the challenges in measuring both aerodynamic and acoustic fields in the same facility. Although measurement access is not an issue for numerical simulations, practical limitations on the simultaneous resolution of the entire unsteady flow field make it essential to adopt a zonal approach that emphasizes a small subset of flow features and test conditions. As a result of such challenges, any strategy to develop validated methodology for physics based aeroacoustic predictions for high-lift devices (even when it involves a hybrid approach that decouples the unsteady aerodynamics in the near field from the acoustic propagation away from the model) must involve a number of compromises as outlined below at several places within this section. Furthermore, it becomes impractical to evaluate the impact of each individual numerical parameter in the context of such simulations, forcing one to rely on a combination of partial, case-by-case assessments and prior experience (depending on the available computational resources) to make judicious choices. 


\section{A. Geometry of High-Lift Configuration and Flow Conditions}

Similar to the 2D computations in [19], the present simulations are based on the $30 \mathrm{P} 30 \mathrm{~N}$ model geometry that was used during the experiments in the Basic Aerodynamic Research Tunnel (BART) at NASA Langley Research Center [22]. The relatively small (28 inch by 40 inch) test section of BART precludes meaningful noise measurements in this facility; however, it is well suited for characterizing the fluid dynamics of the high-lift configuration. The $30 \mathrm{P} 30 \mathrm{~N}$ geometry represents a three-element, high-lift configuration with slat and flap deflections of 30 degrees each (Fig. 2); it has a rich history of previous investigations, including the studies performed in the context of a CFD challenge sponsored by NASA Langley in 1993 [20] as well as additional, follow-up studies as described in Refs. [24, 27-29]. The slat chord and flap chord of the 30P30N model are equal to $15 \%$ and $30 \%$, respectively, of the stowed chord. For the approach configuration of interest in the present study, the slat gap is $2.95 \%$; the flap gap is $1.27 \%$; and the slat and flap overhang settings equal $-2.95 \%$ and $0.25 \%$, respectively. (See Ref. [20] for a definition of the rigging parameters.) The trailing edges of all three elements have a finite thickness. However, for computational purposes, only the slat thickness of $0.36 \mathrm{~mm}$ is resolved in the computational grid. The trailing edges of the wing and the flap were artificially sharpened while preserving the camber of the respective sections.

At the test Mach number of $M=0.17$, the Reynolds number $\operatorname{Re}_{c}$, based on the stowed chord of 18 inches for the BART model, equals 1.7 million. Previous experiments with another 2D high-lift configuration [C.L. Streett, private communication, 1999] had indicated that the measured noise characteristics become relatively insensitive to the Reynolds number parameter for $\mathrm{Re}_{\mathrm{c}} \geq 7.2$ million. (See, also, Ref. [5].) The lower Reynolds number in the BART experiments reflects an essential compromise of the type mentioned at the beginning of this section. A likely aeroacoustic artifact of low Reynolds number high-lift configurations corresponds to prominent narrowband peaks in both nearfield and farfield frequency spectra [3,30]; some of these peaks have been attributed to Rossiter modes of the open cavity flow in the slat cove region. ${ }^{30}$ However, no evidence of narrowband peaks (other than the peak associated with vortex shedding from the slat trailing edge) was found during the present simulations for the BART configuration. The geometry of the BART $30 \mathrm{P} 30 \mathrm{~N}$ model was nominally two-dimensional. A redesigned set of slat brackets provided the required optical access ${ }^{22}$ while minimizing the interference with the overall flow field. However, no special treatment such as boundary layer suction was employed along the side walls of the tunnel. Consequently, even though a quasi-2D flow behavior is expected near the center of the 40 inch test span, the flow field during the experiment is likely to involve finite $3 \mathrm{D}$ effects due to the side walls of the tunnel.

The close proximity of the tunnel walls in the BART facility also implies that the acoustic field within the wind tunnel is greatly influenced by acoustic reflections from the walls. Even though the computational grid becomes significantly coarser in regions away from the test model, effects of acoustic reflections from the tunnel walls were clearly visible in the 2D computations in Ref. [19]. To enable the simulation database to be used towards acoustic computations in a follow-up study, the 3D computations are carried out with the $30 \mathrm{P} 30 \mathrm{~N}$ model in a free-flight configuration. To facilitate a simultaneous comparison with the unsteady flow field measured during the BART experiment, the angle of attack for the free-flight configuration has been adjusted to mimic the measured mean pressure distributions along the slat lower surface and the wing leading edge. Based on 2D, steady state calculations at various angles of attack, an angle of attack $\alpha=5.5$ degrees in the free-air calculation was determined to provide an approximate match with the local Cp distribution based on both BART measurements and a 2D RANS solution for the tunnel installed model at a 4 degree angle of attack (Fig. 3).

\section{B. Computational Grid}

The computational grid for the 3D simulations is adapted from the earlier 2D grid in Ref. [19] by extending the outer boundaries of the computational domain (to simulate free-flight configuration), but reducing the grid density away from the slat cove region (in order to make the $3 \mathrm{D}$ computations more tractable). The outer boundaries are positioned at a minimum of 10 chord lengths away from the center of the model. A uniform grid is used along the spanwise direction (z). Based on prior experience with 2D, time accurate simulations for this (and other) configurations, the spanwise width of the computation domain is chosen to be $1 \mathrm{inch}$, i.e., $5.6 \%$ of the stowed chord, $37.3 \%$ of the slat chord, or $2.5 \%$ of the overall span of the BART model.

It is worth pointing out that the spanwise length scale is likely to vary considerably over the computational domain. Not only does one expect the spanwise length scale for the trailing edge shedding to be different from that in the slat cove region, but this length scale may also vary along the length of the slat cove shear layer. 
Unfortunately, for practical reasons, we are constrained to use a uniform grid spacing over a fixed width domain along the spanwise direction.

A comprehensive study of grid convergence is currently not feasible for time accurate simulations of high-lift configurations. However, given the emphasis on evaluating the improvements in the accuracy of the captured slat cove structures via the change from 2D to 3D computations, two separate spanwise resolutions have been employed during the present simulations. In grid S1, the spanwise domain is discretized with 33 points, the minimum resolution deemed worth considering for the 3D simulations. Grid S2 involves 65 spanwise points over the same domain, i.e., provides twice the spanwise resolution in comparison with grid S1; in addition, a few minor enhancements were made in this case to provide an improved $x$-y resolution immediately downstream of the slat trailing edge. In the case of the grid S1, the planar grid (i.e., in the $x-y$ plane) consists of $400.4 \mathrm{~K}$ grid points, for a total of 13.21 million points across the overall computational domain. The improved resolution of the slat wake in grid S2 led to an approximately 5\% increase in the planar grid count, yielding a total of 27.27 million points over the same domain. The planar grid density may be compared with the recommended minimum values of $50 \mathrm{~K}$ grid points for accurate predictions of surface pressures and $100 \mathrm{~K}$ to $200 \mathrm{~K}$ grid points for accurate predictions of velocity profiles using stationary RANS computations [21]. The wall normal spacings for both S1 and S2 are the same, being close to unity or less (in terms of wall units) over the slat and the flap, and less than 1.6 wall units over the main element. Overall, the planar grid is designed to resolve unsteady flow structures within the slat cove and the near wake of the slat; however, it does not resolve the boundary layer turbulence, or the unsteady features within the far wake of the slat and the main and flap wakes, the main element cove or, as seen later, the separated flow region over the aft portion of the flap upper surface.

The portion of the grid in the immediate vicinity of the slat surface, including the slat cove, accounts for approximately one half of the total number of grid points. The planar grid was designed to accommodate, as much as possible, the considerable excursions in the instantaneous locations of the slat cove vorticity structures (i.e., did not stretch rapidly away from the mean trajectory of the slat mixing layer). A posteriori inspection of the computed solution indicates that there are more than 100 points across the mean vorticity (or maximum slope) thickness of the slat cove mixing layer at a sample location corresponding to 82 percent of the trajectory length from the slat cusp to the reattachment location (i.e., somewhat upstream of the flow reattachment location). In comparison, the grid spacing along the other coordinate direction within the $\mathrm{x}-\mathrm{y}$ plane (which is aligned approximately with the direction of the mean mixing layer flow) is nearly 5 times larger. For grid S2, the spanwise grid spacing at this location is slightly over twice as large in comparison with the local grid spacing along the mixing layer. Close to the cusp, the grid spacings along the two coordinate directions within the $x-y$ plane become comparable with each other and the resolution across the shear layer is considerably less fine as that in the downstream region.

\section{Simulation Methodology}

Similar to the 2D computations in Ref. [19], version 6 of the CFL3D flow solver ${ }^{31}$ developed at NASA Langley Research Center is used for the 3D simulations. This version solves the thin layer form of the compressible NavierStokes equations using a finite volume discretization; the viscous fluxes in all three grid coordinates were retained during the present simulations. The present simulations employed the third order upwind scheme with flux difference splitting, which has been shown to provide second order spatial accuracy in previous applications of CFL3D. Recent work by our colleagues at NASA Langley Research Center $^{32}$ has indicated that even second order flow solvers, which were originally developed for steady state CFD calculations, can provide reasonable predictions for unsteady separated flows such as bluff body wakes.

The simulations were performed using characteristic boundary conditions along the far-field boundaries in the $\mathrm{x}-\mathrm{y}$ plane, except for extrapolation from the interior at the downstream boundary; and periodic boundary conditions across the spanwise boundaries of the computational domain. No-slip conditions are imposed at the solid surfaces, along with an adiabatic wall thermal boundary condition.

The two-equation Shear Stress Transport (SST) model of Menter $^{33}$ is used to capture the mean flow behavior in regions away from the slat cove region, whereas the turbulence production term in the turbulence transport equations was switched off within the cove region to eliminate the excessive diffusive effects of the turbulence model on the resolved unsteady flow structures. The grid S2 also allowed the near wake flow behind the slat trailing edge to be treated as pseudo-laminar, analogous to the slat cove region. 
The assumed laminar origin for the slat cusp shear layer is justified on the basis of the relatively small Reynolds number for the unswept model, coupled with first, a short boundary layer development length between the slat stagnation line and the cusp and, second, the strong favorable pressure gradient over that distance. ${ }^{15}$ Indeed, the computed boundary layer profile just ahead of the slat cusp is laminar in shape and has a momentum thickness Reynolds number of $\operatorname{Re}_{\theta} \approx 131$, which is subcritical even in the case of a (more unstable) zero pressure gradient boundary layer. Because the pressure gradient along the upper surface of the slat is generally favorable (with the exception of a rather weak suction peak), the boundary layer flow in this region is expected to be mostly laminar. To maintain consistency with the previous $2 \mathrm{D}$ simulations, ${ }^{15,16,19}$ however, the flow everywhere else but the slat cove region was assumed to be fully turbulent, allowing the transition locations along all three elements to be determined by the SST turbulence model. We note in passing that the 3D calculations used a more recent implementation of flux limiting within CFL3D, which has been designed to provide more consistent results for multi-block domains [R.T. Biedron, private communication, 2003]. This consistency was deemed especially important in the 3D case, because the slat cove region had to be divided into a number of separate grid zones in order to permit parallel runs on sufficiently large number of processors.

To provide an appropriate starting condition for the $3 \mathrm{D}$, time accurate simulations of slat cove unsteadiness, steady state calculations for the $30 \mathrm{P} 30 \mathrm{~N}$ configuration were performed for an $\mathrm{AOA}$ of 5.5 degrees in free flight configuration. Because of the fine spatial resolution within the cove region, the steady state calculations tend to develop unsteady vortex structures in the slat shear layer despite the fact that the flow was assumed to be fully turbulent throughout the domain. Accordingly, the steady state calculations (based on a constant local CFL number of 1.0) were terminated before multiple vortices developed within the cove region, switching to a time accurate mode with a pseudo-laminar slat cove region. Our experience with 2D, time accurate calculations of this and similar high-lift flows suggests that the above starting sequence generally provides a reasonable starting flow for the time accurate computations, avoiding some of the unphysical flow features resulting from some other modes of startup.

The time step used during the unsteady simulation with grid S1 corresponds to a sampling frequency of 0.88 $\mathrm{MHz}$, i.e., a temporal resolution of 100 time steps over one period of an $8.8 \mathrm{kHz}$ signal. The time step used for grid $\mathrm{S} 2$ is one half of that used for grid S1. This temporal resolution is deemed sufficient across the expected spectrum of the slat cove unsteadiness and is also just adequate to resolve the fundamental frequency of vortex shedding from the slat trailing edge (and its first harmonic in case of Grid S2 simulation). The flow through (i.e., convective) time scale based on the slat chord and the free-stream speed corresponds to about 1070 and 2140 time steps, respectively, for the two grids. Time advancement is based on a second order implicit scheme, used in conjunction with the dual time stepping method [31]; fifteen subiterations at a fixed local CFL number are used during each time step during the computation on grid S1, whereas twenty subiterations have been used for the grid S2 simulation. The effect of time step and the number of subiterations was evaluated over a limited duration during the grid S2 simulation. Variations in both integral forces and instantaneous vorticity structures were monitored. Vortices shed from the trailing edge of the slat displayed some sensitivity to temporal discretization parameters, which was to be expected because of their relatively higher frequency. However, the slower slat cove dynamics based on the abovementioned values of temporal simulation parameters agreed well with that predicted (over a short duration) with one half the nominal time step for this grid (and the same number of subiterations over each step) or 1.5 times the number of subiterations over the same time step. No doubt, a more thorough (and longer duration) study of both spatial and temporal convergence is desirable; however, it was beyond the scope of the present project. To the extent that the effects of the different numerical approximations are uncoupled from each other (or loosely coupled, at worst), an a posteriori comparison between the relevant predictions from the grid S1 and grid S2 simulations would provide an increased confidence into the robustness of the numerical predictions.

To accelerate the development of 3D vorticity structures, random 3D, time dependent forcing was applied over a short duration across a suction/blowing slot just ahead of slat cusp. The forcing characteristics were similar, but not identical, between the two simulations. Results show that the 3D fluctuations are self-sustaining in both cases and, moreover, exert a profound influence on the unsteady dynamics of the slat cove region. (Similar effects of local 3D dynamics on other aerodynamic flows have been reported in Ref. [34].) Because the incoming boundary layer is laminar, the possibility that the effects of initial flow history persist for time scales larger than the duration of the computation cannot be discounted a priori. Thus, the numerical simulations were run over a long overall duration that consisted of at least 37 slat flow-through time units in the case of grid S1 and 18 units for grid S2 in order to minimize the transient effects as well as to reduce the convergence error in the flow statistics accumulated over a subset of that duration. 


\section{Results}

Based on the geometry of the slat cove flow, we expect it to share common features with related canonical flows such as a splitter plate mixing layer (without the splitter-plate wake effects) and the flow past a backward facing step (with a large step height relative to boundary layer thickness), with likely modifications due to the proximity of the slat trailing edge and the flow acceleration through the gap between the slat and the main wing. Certain similarities with a blown flap configuration are also possible. Accordingly, we will compare our numerical findings with both the BART measurements on the $30 \mathrm{P} 30 \mathrm{~N}$ configuration and the rich database available for the above canonical flows. The aim here is to build additional confidence into the accuracy of the computed solution, as well as to understand the underlying physical mechanisms governing the dynamics of the slat cove flow.

\section{A. Instantaneous Flow-Field: 3D vs. 2D Predictions}

We begin with an inspection of the instantaneous vortical structures associated with the unsteady slat cove flow. Figure 4(a) presents selected isosurfaces of instantaneous spanwise vorticity at a typical instant in time. The isosurfaces corresponding to both positive and negative spanwise vorticity $\left(\omega_{z} \mathrm{c} / \mathrm{U}_{\infty}= \pm 265\right)$ are shown in yellow and cyan shades, respectively. The shear layer seems to roll up into discrete spanwise vortices almost immediately downstream of the cusp. The quasi-2D vortices soon develop spanwise deformations that amplify with downstream distance, resulting in predominantly $3 \mathrm{D}$ vorticity structures in the downstream region. The transition to threedimensionality, which is analogous to that in the previous studies of splitter plate shear layers, takes place well upstream of the reattachment location just upstream of the slat trailing edge. The nature of 3D vorticity structures within the cove is similar for both grid S1 and grid S2.

The strong three-dimensionality of the vorticity structures is consistent with a number of previous measurements carried out for a splitter plate mixing layer with a zero inviscid pressure gradient [35-38]. Measurements by Chandrsuda et al. [35] have shown that the free-stream turbulence in typical wind tunnel facilities and/or technological applications is often adequate to induce a 3D breakdown of the spanwise vortex mode of mixing-layer instability. While no free-stream turbulence was introduced in the computations, an analogous forcing is provided by the unsteady structures within the recirculation zone. One of the two-stream mixing layer experiments in [35] simulated the flow past a backward facing step by turning off the secondary stream and, indeed, it was observed that the upstream feedback due to entrained turbulence resulted in the vorticity structures becoming strongly three-dimensional almost immediately after their first appearance.

Ref. [35] attributed the dominant cause for the onset of three-dimensionality to helical pairing between the primary vorticity structures. The occurrence of helical pairing has also been noted in other experiments and computational simulations of 3D mixing layers $[39,40]$. The computed dynamics of the vorticity structures within the slat cove is too random to display the regular pattern of helical pairing, but isosurfaces of vorticity magnitude frequently indicate what appears to be localized 3D pairing of mixing layer vortices (Fig. 4(b)). We note that a combination of limited spanwise resolution and the low Reynolds number of the flow may contribute to a somewhat limited 3D mixing transition in the present simulation.

As a result of the mixing layer evolution, the vorticity structures approaching the reattachment region are strongly three-dimensional in nature. Figures 4(a) and 4(c) suggest that a significant fraction of these structures are convected past the slat trailing edge; however, the remaining structures get trapped, at least temporarily, within the recirculation zone and are convected back to the cusp. The convection of the $3 \mathrm{D}$, trapped mixing layer vortices induces unsteady eruptions of secondary vorticity from the recirculating boundary-layer flow along the slat surface, and those are manifested in the form of the cyan colored vorticity blobs in the middle of the recirculation zone. Consequently, the secondary vorticity is fully $3 \mathrm{D}$ in nature, i.e., does not include any quasi-2D spanwise rollers (unlike the mixing-layer vortices with positive vorticity). In Fig. 4(a), the secondary vortex activity at the selected vortex strength is somewhat weaker in comparison with the yellow trapped vortices derived from the mixing layer. The sustained, random forcing associated with the recirculating vortices of both signs may account, at least partially, for the rapid 3D deformation of the spanwise rollers formed near the slat cusp.

A comparison between the instantaneous spanwise vorticity distributions across a spanwise cut through the computational domain exemplifies the differences between 3D and 2D predictions based on the same pseudo- 
laminar modeling of the slat cove region (Figs. 5 and 6, respectively). For reference, we have also included similar images based on the planar PIV data from Ref. [22] in Fig. 7. The PIV measurements indicate a regular train of vorticity structures along the slat shear layer; there is occasional entrainment of the vorticity structures into the recirculation zone (cove state I in Fig. 7), however a majority of vortices are simply convected past the trailing edge (cove state II in Fig. 7). This behavior is also representative of the measurements reported in [23] for a different high-lift configuration. The 3D predictions in Fig. 5 resemble the planar PIV data [22], indicating both states of the measured cove flow field; however, the role of recirculating vortices may be somewhat overemphasized within the narrow domain simulation due to its lack of large-scale 3D effects. As discussed in [19], the vorticity structures in the 2D computation are initially somewhat similar to those seen during the PIV measurements; however, the vortex dynamics becomes increasingly chaotic over time, contributing to an excessively diffused mean vorticity pattern as seen later. We note that that the solutions in [19] for the tunnel installed configuration were still evolving in time. Solutions for the free-flight configuration in Fig. 6 have been run for more than 80 units of slat flow through times, with the average in Fig. 6 accumulated over the last 18 time units. We note in passing that a separate set of unpublished computations had indicated a similarly chaotic slat cove dynamics in the 2D case despite such modifications as either a partial restoration of turbulence production within a thin region adjacent to the lower slat surface or a 2D implementation of a RANS-LES hybrid model. Based on the description of the vortical structures in the $3 \mathrm{D}$ solution, the above deficiency of the $2 \mathrm{D}$ predictions might be attributed to the absence of 3D breakdown prior to the reattachment, which leads to correspondingly strong, spanwise coherent vortices even within the recirculation zone of the $2 \mathrm{D}$ solution.

The 3D vorticity structures convecting towards the slat trailing edge get distorted by the local mean flow strain. Specifically, the stretching of streamwise vorticity (i.e., vorticity along the local mean flow direction) as a result of the mean flow acceleration behind the reattachment leads to streamwise elongated vorticity structures convecting underneath the slat trailing edge. We recall that, based on an earlier set of measurements in BART with the same 30P30N model, Ref. [29] had reported observing some PIV images that were "indicative of a streamwise vortex emanating from the slat cove." The convected slat cove structures interact with the smaller scale vortices shed behind the relatively thin slat trailing edge $(0.36 \mathrm{~mm}$ in thickness), as well as perturbing the wing boundary layer beneath them.

Fig. 4(c) suggests that the perturbations in the wing boundary layer resemble streamwise streaks with a relatively large spanwise length scale (two streaks occupy most of the spanwise domain in Fig. 4(c)). The boundarylayer perturbations over a significant portion of the upper surface are dominated by the streamwise velocity fluctuation u'. The peak u' fluctuation occurs within the main part of the boundary layer (as against the near-wall peak in turbulent kinetic energy in a turbulent boundary layer) and, moreover, increases in magnitude along the chordwise direction over nearly one third of the main element chord. These characteristics of the perturbation field in the main element boundary layer resemble the Klebanoff modes in a laminar boundary layer interacting with freestream turbulence [41]. Although Klebanoff modes in a laminar boundary layer have been suspected of acting as catalysts during the laminar-to-turbulent transition process, the streamwise streaks in the main boundary layer (even if they were to be observed in an actual flow field) are not likely to have the same physical significance as the Klebanoff modes. This is because the main element boundary layer probably transitions near the suction peak of the static pressure distribution, i.e., well upstream of the computed peak in u' fluctuations. The computed behavior of boundary-layer perturbations does, however, indicate a potentially significant interaction between the slat cove unsteadiness and the turbulent boundary layer over the main element.

\section{B. Time Averaged Flow}

Having looked at the instantaneous flow structures in the 3D slat cove solution, we next examine the time averaged flow characteristics and compare the numerical predictions with the measured PIV data.

\section{Mean Flow Streamlines}

Mean flow streamlines based on averaging in both time and span are shown in Fig. 8. For grid S1 simulation, the mean flow data has been averaged over the last 13000 time steps (i.e., more than 12 slat flow-through units); the grid S2 results (not shown here) derived from an average over the last 17000 time steps (i.e., nearly 8 slat flowthrough units) are very similar to those based on the grid S1 simulation. The predicted distance between the shear layer reattachment location and the slat trailing edge is equal to $6.94 \mathrm{~mm}$ and $7.25 \mathrm{~mm}$ based on the grid S1 and grid $\mathrm{S} 2$ simulations, respectively. The same distance based on BART PIV data is equal to $6.77 \mathrm{~mm}$ for the $\alpha=4$ degree 
case targeted during the simulations, and $8.54 \mathrm{~mm}$ at $\alpha=6$ degrees. In comparison, the mean reattachment location predicted by the 2D computations is further away from the trailing edge (at approximately $10.3 \mathrm{~mm}$ ) as a result of the curving of the shear layer trajectory towards the cusp as the reattachment location is approached. For future reference, we note that the length of the computed shear layer trajectory from the cusp up to the reattachment location is approximately $55 \mathrm{~mm}$, which corresponds to a development length of $\mathrm{s} / \theta_{\mathrm{i}} \approx 1590$ relative to the boundary layer momentum thickness near the cusp (s being the arc length along the trajectory, such that $\mathrm{s}_{\max } \approx 55 \mathrm{~mm}$ ). The trajectory shape in 3D simulations is almost circular (except in the vicinity of the reattachment location), with an approximate radius of curvature equal to $29 \mathrm{~mm}$.

The regions of flow separation along the aft of the flap suction surface and the cove region near the main element trailing edge are also expected to sustain significant levels of unsteadiness; however, given the focus on slat noise in the present study, the computational grid in the other regions does not provide adequate spatial resolution of the anticipated unsteady structures. The occurrence of flow separation along the flap can also be inferred from the measured static pressure distribution in [22]; however, we did not investigate to what degree the extent of flow separation is influenced by the relatively low Reynolds number for the BART 30P30N model. Experimental observations of analogous flow separation over the slat upper surface of a high-lift configuration were described in Ref. [42], whereas Ref. [43] presents measurements of unsteadiness in the flap cove underneath the main element.

\section{Cove velocities}

A comparison between the computed and measured mean shear-layer profiles at selected stations (indicated in Fig. 9(a)) along the aft part of the shear layer trajectory is shown in Figs. 9(b) and 9(c). Computed results in Fig 9(b) are based on grid S1 simulation, whereas those in Fig. 9(c) are based on grid S2 simulation. In general, the velocity profiles are not perfectly antisymmetric about the centerline position, both because of the moderate streamwise curvature of the shear layer trajectory and the presence of the recirculation zone on the side of the slower stream. In Figs. 9(b)-(c), the maximum slope of the computed velocity profiles agrees well with the measured data; however, computations underpredict the velocity differential across the mixing layer. We speculate that the larger magnitude of the predicted velocities on the recirculation side is likely to be caused by the absence of large-scale 3D effects from the computation, which precludes a mean spanwise advection of the vorticity structures within the recirculation zone, and (to a smaller degree) by the lack of physical subgrid scale dissipation in the simulations contributing to somewhat stronger large scale vortices. Interestingly enough, the recirculation speeds based on the grid S1 simulation are in better agreement with the PIV data than the corresponding predictions based on the grid S2 simulation. The reasons behind the discrepancy between the two simulations are not clear. They may be caused by the differences in numerical parameters (spanwise grid and temporal discretization parameters), or be an artifact of the somewhat longer duration of averaging enabled by the smaller number of grid points and a larger time step during the grid S1 simulation, or even be related to the facts that mixing layer statistics can vary significantly across individual spanwise locations $[44,45]$ and that the PIV data was acquired at a single location. Nonetheless, the level of agreement between the three sets of profiles is quite encouraging.

Variations in the vorticity thickness $\delta_{\omega}$ of the mixing layer along its trajectory can also be estimated using the velocity profiles averaged in time and span. Within the middle portion of the shear layer trajectory (between approximately $10 \%$ to $60 \%$ locations from the cusp to the mean reattachment point), the estimated growth rate corresponds to $\mathrm{d} \delta_{\omega} / \mathrm{ds} \approx 0.1$. The external mixing layer velocity is nearly constant over the above range of locations (along with a nearly constant pressure except for a region of favorable pressure gradient closer to the cusp); however, due to the variations in speed along the recirculation side, the velocity ratio for the slat mixing layer varies over the range of $(0.13,0.25)$ and $(0.2,0.3)$ for the grid S1 and grid S2 simulations, respectively. The nearly constant, stabilizing curvature of the shear layer may contribute to a slightly reduced spreading rate relative to straight mixing layers, especially along the aft region of the shear layer trajectory where the layer thickness is larger. Overall, the predicted spreading of the slat cove shear layer appears to be in line with both the BART experiment and the typical range of spreading rates for a conventional mixing layer flow.

\section{Cove Vorticity}

As may be expected from the description of the instantaneous vorticity structures, the differences between the 3D and 2D solutions become further apparent from a comparison between the respective time averaged spanwise vorticity distributions (Fig. 10). Because of the chaotic vortex dynamics in the $2 \mathrm{D}$ case, the corresponding time averaged vorticity field is considerably more diffuse compared to that from the 3D computation, which shows a more well-defined mean shear layer similar to the PIV data from Ref. [22]. Furthermore, an overly strong signature 
of the trapped vortices convecting back to the slat cusp is also obvious in the 2D results. (see, also, Fig. 9(a) from Ref. [19].) We note, however, that in contrast to the 3D results, the PIV data indicates a relatively thicker shear layer close to the cusp. The reason behind this discrepancy is attributed to a lack of sufficient spatial resolution in the PIV data in that region. The resolution of the processed PIV data corresponds to $0.7 \mathrm{~mm}$ at best and is considerably worse near the cusp region [L. Jenkins, private communication, 2005], which is inadequate to resolve the large velocity gradients associated with a thin mixing layer. This discrepancy becomes even more obvious during the subsequent comparison of the turbulent kinetic energy distribution, wherein we include the PIV data based on a smaller field of view to resolve some of the discrepancy between computational predictions and the PIV data based on the larger field of view (Figs. 7, 9, and 10).

\section{Fluctuation Statistics}

\section{TKE Distribution}

Contours of the predicted and measured distributions of 2D turbulent kinetic energy (TKE), $\frac{1}{2}\left(\left\langle u^{\prime} u^{\prime}\right\rangle+\left\langle v^{\prime} v^{\prime}\right\rangle\right)$,

are shown in Figs. 11(a) through 11(c). The "hammer" shape of the PIV based contours of TKE distribution near the reattachment location is also captured within the 3D simulation; however, the computations do indicate somewhat higher levels of fluctuation energy within the recirculation zone. In contrast, the nature of 2D TKE distribution is quite different in the 2D computation (Fig. 11(a)), which shows unrealistically high TKE levels within the recirculation zone. Indeed, the discrepancies related to the TKE distribution had been cited in our earlier work ${ }^{16}$ as one of the major weaknesses of the 2D predictions based on the same pseudo-laminar treatment of the slat cove region. The qualitative agreement between the 3D solution and the PIV data makes the TKE distribution based on the time accurate calculations more consistent with both experiments ${ }^{22,23}$ and the predictions based on stationary RANS calculations with a two-equation turbulence model $^{16}$.

The peak velocity fluctuations across the slat mixing layer are plotted in Fig. 12 against the distance s from the slat cusp along the mean shear-layer trajectory. The initial overshoot in the peak turbulence intensity $\mathrm{Tu}_{2 \mathrm{D}} \equiv$ $\left[\frac{1}{2}\left(\left\langle u^{\prime} u^{\prime}\right\rangle+\left\langle v^{\prime} v^{\prime}\right\rangle\right)\right]^{1 / 2}$ has also been observed during previous measurements of mixing-layer evolution from an initially laminar boundary layer [46, 47], where it was attributed to a laminar-turbulent transition in the separated flow. We note that the Reynolds number for the BART configuration is fairly low (e.g., the local Reynolds number based on the vorticity thickness at $\mathrm{s} / \mathrm{s}_{\max }=60 \%$ is approximately 14300) and, quite possibly, is marginal for a sustained fine-scale motion based on the data quoted in Ref. [47]. On the other hand, the latter data pertains to conventional, splitter plate mixing layers and the sustained forcing due to recirculating vortices in the present case may result in significantly reduced values of threshold Reynolds numbers for sustained small-scale motion. Of course, it is not obvious whether or not the spatial grids used during the present simulations can resolve the details of the transition process. Therefore, it is interesting that the associated behavior of the turbulent kinetic energy (which should be controlled by the resolved eddies) is reflected within the simulation results.

The magnitudes of peak shear-layer fluctuations (Fig. 12(b)) upstream of the reattachment region fall within the expected range of values based on the prior measurements of splitter plate mixing layers [47]. We also note that the evolution of $\mathrm{Tu}_{2 \mathrm{D}}$ along its peak trajectory has a second maximum just upstream of the mean reattachment location. Increased turbulence energy just upstream of the reattachment location has also been noted in earlier experiments on slat cove unsteadiness $[22,48]$. While the planar velocity fluctuations ( $\mathrm{u}^{\prime}$ and $\mathrm{v}^{\prime}$ ) decay rapidly as the reattachment location is approached, the spanwise velocity fluctuations do not decay as a result of the accompanying changes in the mean flow velocities in the $x-y$ plane. In fact, the peak amplitude of the w' fluctuations continues to increase past the second maximum in $\mathrm{Tu}_{2 \mathrm{D}}$ and decays only in the immediate vicinity of the reattachment location (presumably due to viscous effects). Consequently, the 3D TKE evolution displays two separate peaks near the reattachment location, one near the peak in $\mathrm{Tu}_{2 \mathrm{D}}$ and the other near the peak of the w' curve (Fig. 12).

The planar PIV measurements in Fig. 12(a) can, of course, only capture the first of the above two peaks and, indeed, are quite consistent with the computed evolution of $\mathrm{Tu}_{2 \mathrm{D}}$ in this regard. Within the upstream portion of the shear layer trajectory, however, the measured PIV data based on a larger field of view (f.o.v.) indicates quite significant discrepancies with the numerical predictions. The reason behind this discrepancy is not fully clear; 
however, we attribute the differences, to a large extent, to a lack of sufficient spatial resolution in the larger f.o.v. PIV data near the cusp where the mixing layer is relatively thin. Indeed, a portion of the mixing-layer evolution close to the slat cusp was captured within the smaller f.o.v. PIV data, which seems to capture the fluctuation amplitudes near the predicted overshoot presumably associated with shear-layer transition. Unfortunately, the higher resolution data does not extend sufficiently farther downstream (as to match with the larger field of view data within a finite region of overlap). It is also known that mixing layer flows tend to exhibit significant spanwise variations in the time averaged flow statistics [38, 44, 45, 47] and, therefore, measurements at multiple spanwise locations are necessary to characterize the streamwise evolution of the flow. In view of the above observations, a more complete validation of the predicted TKE evolution will have to await additional measurements of the slat cove flow field.

\section{Pressure Fluctuations}

Pressure fluctuations along the solid surface are known to play an important role in both understanding and predicting the noise generation mechanisms due to flow-surface interactions [49-51]. The computed distribution of root mean square (r.m.s.) pressure fluctuations along the slat surface is shown in Fig. 13. The peak surface pressure fluctuations occur on the lower side in the vicinity of the reattachment location, again highlighting the likely significance of this region during the process of slat noise generation. The predicted amplitude of pressure fluctuations in the present case is higher than the peak r.m.s. fluctuation in $\mathrm{Cp}$ of 0.06 previously measured near the reattachment downstream of a backward facing step [52]. Certainly, a part of the difference in Cp' magnitudes may be related to the inherent differences between the respective flow configurations and the larger than expected spanwise coherence in the computational simulations (see subsection 3. below); however, it will be very useful to have future measurements of slat pressure fluctuations near the reattachment region to help resolve this important issue. Measurements of slat pressure fluctuations have been reported in Ref. [53]; however, the data in Ref. [53] is limited to the frequency spectrum at a microphone location that lies in between the reattachment location and the slat cusp and, hence, cannot be used to infer the peak pressure fluctuation.

We found that the peak pressure fluctuations along the main element surface are concentrated within the leading edge region; but, the amplitude of those fluctuations is weaker than the slat pressure fluctuations near the reattachment location. Because of the flow separation along the upper surface of the flap, significant pressure fluctuations are also likely to occur near the separation location. However, the spatial grid used during the present simulations is not designed to capture the unsteady dynamics in that region.

\section{Spanwise Correlation}

Computed spanwise correlations of the fluctuations at selected slat cove locations are shown in Figs. 14(a)-(c). A scrutiny of the spanwise correlations serves two important purposes, first as an a posteriori measure to assess the adequacy of the spanwise resolution (i.e., grid spacing and domain width) and, second, to provide a glimpse into the likely spanwise distribution of the acoustic sources associated with slat cove unsteadiness. The source distribution has a direct impact on the far-field acoustic intensity, as the latter scales linearly with the spanwise coherence length (i.e., the effective length over which the acoustic source distribution at a given frequency may be assumed to be phase synchronized) when the spanwise extent of the source distribution is sufficiently large. Browand and Troutt [36] had measured the spanwise correlations in the irrotational region of a turbulent mixing layer with a velocity ratio of 0.8 ; they reported that the correlation coefficient dropped to 0.2 at a spanwise separation of approximately $3 \delta_{\omega}$. Jones, Planchon, and Hammersley [54] and Wygnanski (private communication as reported in [35]) quote spanwise integral scales of $0.2-0.3 \mathrm{~s}$ in the irrotational flow on either side of the mixing layer (where $\mathrm{s}$ now denotes the longitudinal coordinate relative to the effective origin of the mixing layer), but only about $0.03 \mathrm{~s}$ within the mixing layer. The latter scale agrees with the measurements by Chandrsuda et al. [35], who report an integral scale of roughly $0.15 \delta_{\omega}$ in the fully turbulent region. They also report measuring smaller but finite spanwise correlations at large separations within the initial region, indicating the presence of 2D spanwise rollers.

Figures 14(a) and 14(b) indicate the spanwise correlation coefficients for vertical velocity fluctuation at selected locations within the slat shear layer, as computed using the data from grid S2 and grid S1 simulations, respectively. Correlation coefficient for the surface pressure fluctuation near the reattachment location (based on grid S2 simulation) is shown in Fig. 14(c). A comparison between Figs. 14(a) and 14(b) shows that the spanwise correlation length based on the coarse spanwise grid is significantly larger than that predicted using the finer spanwise grid; and there are also noticeable differences in the nature of the (weaker) correlation at larger spanwise separations. In both cases, however, the changes in spanwise correlation length across the three stations are smaller than the changes in 
the longitudinal coordinate s. Since the predictions for the grid S1 simulation appear to be statistically converged in general, we tend to attribute the discrepancy between the correlations at smaller $\Delta \mathrm{z}$ to the absence of the smaller spanwise scales from the grid S1 simulation which may contribute to the destruction of spanwise coherence. A preliminary comparison suggests that the correlation "length" based on the grid S2 data is also larger in comparison with the abovementioned measurements for the splitter plate shear layer; this observation would be consistent with the previous remarks concerning the somewhat limited spanwise resolution even in grid S2. Clearly, additional simulations and experimental measurements would help resolve this yet another important issue.

Guo and Joshi [10] have pointed out that the observed $\mathrm{M}^{5}$ scaling of the farfield intensity of slat noise may be interpreted either in terms of edge scattering or via a distributed acoustic source that is coherent over spanwise distances in excess of the acoustic wavelength at the relevant frequencies. The predominance of 3D vorticity structures within the slat cove region, coupled with the acoustically compact spanwise correlation of the slat cove fluctuations in the simulation data, indicates that the cause behind the observed Mach number scaling of slat noise is less likely to involve an acoustic source with an extended spanwise coherence.

\section{Frequency Spectra}

To gain further insights into the nature of fluctuations associated with the unsteady slat cove flow, we next examine the frequency spectra of velocity fluctuations at selected locations along the mean trajectory of the slat cusp mixing layer (Fig. 15(a)). For each of the curves plotted in Fig. 15(a), the probe position is chosen to be in close vicinity of the locations of peak mean vorticity and peak TKE at the corresponding station along the trajectory. The frequency spectrum at the probe location closer to the cusp indicates a peak near 9 to $10 \mathrm{kHz}$, which fades as the mean shear layer thickens. The frequency spectra at the other two locations indicate a limited extent of energy cascade corresponding to an approximately $-5 / 3$ power law roll-off. The presence of an equilibrium subrange in the computed spectra is somewhat surprising as one might have expected the spatial wave-number cutoff to be just beyond the energy containing scales, at best.

Frequency spectra across a slat wake profile approximately $0.5 \mathrm{~mm}$ downstream of the slat trailing edge are shown in Fig. 15(b). With the frequency parameter plotted on a logarithmic scale and the ordinate corresponding to frequency parameters times the power spectral density, the area under the plotted curve within a specified range of frequencies is proportional to contribution from those frequencies to the total energy in the signal. The transverse probe positions are selected to be close to the local peak of fluctuation amplitude for that particular velocity component. For the most part, the spectra for all three velocity components are dominated by the high-frequency peak associated with vortex shedding from the finite thickness trailing edge. However, the peak w' fluctuations (which occur further below the region of peak shear associated with the slat wake and are likely to represent the effect of vorticity structures convecting across the slat gap) show a distinct peak in the low frequency region (1-4 $\mathrm{kHz}$ ), which overlaps with the anticipated range of peak frequencies associated with the broadband slat noise. The role of the spanwise velocity fluctuations in the noise generation process remains to be determined.

Finally, the frequency spectrum of pressure fluctuations near the reattachment location is shown in Fig. 15(c). It is clearly dominated by the lower frequencies that fall within $1.5-4 \mathrm{kHz}$, with a broad peak near $2.2 \mathrm{kHz}$. A rudimentary analysis, based on the estimates of local mean vorticity thickness, indicates the natural frequencies of the shear layer to fall within the $1.5-5 \mathrm{kHz}$ frequency band along the middle portion of the shear layer trajectory. These frequencies are consistent with the recorded pressure fluctuations near the reattachment location (Fig. 15(c)) and indicate a connection between the most unstable shear-layer disturbances and high amplitude surface pressure fluctuations.

\section{Acoustic Implications}

A study of farfield acoustic radiation from the slat cove region using 3D nearfield predictions (and its comparison with purely 2D calculations) is beyond the scope of the present paper; however, some preliminary insights concerning slat noise characteristics can be easily gleamed from the present computations. Of course, in interpreting these results, one must bear in mind the limitations of present computations due to their narrow (and yet marginally resolved) spanwise domain and periodic spanwise boundary conditions. The frequency spectra of the computed pressure fluctuations at selected probe locations at distances between 1 to 2 slat chords away from the slat 
trailing edge are shown in Fig. 16(a). Probes below the slat are shifted towards the rear, whereas those above the slat are shifted to the forward arc. The acoustic spectra across the range of frequencies plotted in the figure closely resemble the spectrum of pressure fluctuations near the reattachment location. Additional analysis of the computed database will include an analysis of correlations between the nearfield and the acoustic signals, in order to establish the potential causal relationships between the two. The peak Strouhal number of the acoustic spectra in Fig. 16(a) lies within the range of $\mathrm{St}=1-3$, similar to the previous measurements of subscale high-lift configurations [8]. The roll-off in the computed acoustic spectra beyond the above peak (up to about $14 \mathrm{kHz}$ ) corresponds to approximately $\mathrm{f}^{2}$, lending partial support to the analytical model by Guo [51].

Dobrzynski and Pott-Pollenske [8] had modeled the broadband slat noise source as an acoustic dipole normal to the slat surface near the trailing edge (Fig. 16(b)). The directivity of the computed nearfield fluctuations resembles this behavior (Fig. 16(c)) although, of course, far-field predictions are necessary to enable definitive comparisons with the above prediction.

\section{Summary and Discussion}

In this paper, we have presented $3 \mathrm{D}$, time accurate simulations of flow dynamics near the slat cove of an unswept, 3-element high-lift configuration, for flow conditions that are relevant to aircraft approach for landing. The favorable comparison with the planar PIV data and previous measurements of splitter plate mixing layers represents a substantial improvement over the previous $2 \mathrm{D}$ computations, revealing the importance of $3 \mathrm{D}$ fluctuations in determining the unsteady dynamics within the cove region. The computed database could play a useful role in discovering and ranking the physical mechanisms contributing to the generation of slat noise. The present analysis indicates that the reattachment region on the lower slat surface might play a key role in slat noise generation, both because of the relatively high local turbulence intensity/surface pressure fluctuations and its proximity to both regions of mean flow strain and boundary inhomogeneities such as the slat trailing edge and the narrow gap between the slat and the main element. The effects of mean flow strain have been addressed in a recent work [55] that examines a model problem involving the interaction between an array of vortices and the irrotational mean flow due to a circular cylinder.

The computed acoustic characteristics (in terms of the frequency spectrum and spatial distribution) within short distances from the slat resemble the previously reported, subscale measurements of slat noise. Observed similarities between the dynamics of the slat shear layer and the conventional mixing layer downstream of a splitter plate suggest that the established database and models for space time correlations for the latter (which have already been used for jet noise predictions) may be used as input to reduced order models for acoustic predictions of leading edge slats [56]. In addition to clarifying some of the physics associated with the aeroacoustics of the slat cove, the present work has also raised some specific questions concerning the pressure fluctuations near the reattachment location and the spanwise correlations of slat cove fluctuations, both of which would be useful targets for future simulations and experiments. Currently, we are pursuing wind tunnel measurements related to the 3D aspects of slat cove velocity fluctuations, along with additional simulations that address some of the gaps in the work described herein. A more thorough validation of the computational methodology in this manner will provide the desired foundation for (limited) parametric studies related to the effects of the Reynolds number, angle of attack, and slat deflection and sweep angles.

\section{Acknowledgments}

The authors gratefully acknowledge the contributions of various colleagues at NASA Langley Research Center, especially Mr. Luther Jenkins, Cathy McGinley, and Dan Neuhart of the Flow Physics and Control Branch and Drs. David Lockard, Bart Singer, Jay Casper, Craig Streett, Robert Rubinstein, Robert Biedron and Chris Rumsey from the Computational Aerosciences Branch. Luther Jenkins kindly provided the processed PIV data for comparison with the numerical results. MC acknowledges useful technical conversations with Prof. Geoffrey Lilley (Univ. of Southampton, U.K.), Prof. Ugo Piomelli (Univ. of Maryland), and Dr. Alan Cain (Innovative Technology Applications Company). Thanks are also due to Mr. M.R. Wiese of the Analytical Services and Materials, Inc. for generating the grids used during the computations.

\section{References}

1. Hayes, J.A., Horne, W.C., Soderman, P.T., and Bent, P.H., "Airframe Noise Characteristics of a 4.7\% Scale DC10 Model," AIAA Paper 97-1594, May 1997. 
2. Storms, B.L., Ross, J.C., Horne, W.C., Hayes, J.A., Dougherty, R.P., Underbrink, J.R., Scharpf, D.F., and Moriarty, P.J., "An Aeroacoustic Study of an Unswept Wing with a Three-Dimensional High Lift System," NASA TM 112222, Feb. 1998.

3. Dobrzynski, W., Nagakura, K., Gehlhar, B., and Buschbaum, A., "Airframe Noise Studies on Wings with Deployed High-Lift Devices," AIAA Paper 98-2337, 1998.

4. Storms, B.L., Hayes, J.A., Moriarty, P.J., and Ross, J.C., "Aeroacoustic Measurements of Slat Noise on a ThreeDimensional High-Lift System,” AIAA Paper 99-1957, May 1999.

5. Choudhari, M., Lockard, D.P., Macaraeg, M.G., Singer, B. A., Streett, C.L., Neubert, G.R., Stoker, R.W., Underbrink, J.R., Berkman, M.E., Khorrami, M.R., and Sadowski, S.S., "Aeroacoustic Experiments in the Langley Low-Turbulence Pressure Tunnel,” NASA/TM 2002-211432, Feb. 2002.

6. Mendoza, F. and Brooks, T.F., "Aeroacoustic Measurements of a Wing/Slat Model," AIAA Paper 2002-2604, 2002.

7. Chow, L.C., Mau, K., and Remy, H., "Landing Gears and High Lift Devices Airframe Noise research," AIAA Paper 2002-2408, June 2002.

8. Dobrzynski, W. and Pott-Pollenske, M., "Slat Noise Source Studies for Farfield Noise Prediction," AIAA Paper 2001-2158, 2001.

9. Guo, Y.-P., Yamamoto, K.J. and Stoker, R.W. "Component Based Empirical Model for High-Lift System Noise Prediction,” J. Aircraft, Vol. 40, No. 5, pp. 914-922, 2003.

10. Guo, Y.-P., and Joshi, M., "Noise Characteristics of Aircraft High-Lift Systems," AIAA J., Vol. 41, No. 7, pp. 1247-1256, 2003.

11. Lockard, D.P., and Lilley, G.M., “The Airframe Noise Reduction Challenge,” NASA/TM-2004-213013, 2004.

12. Khorrami, M.R., Berkman, M.E., and Choudhari, M., "Unsteady Flow Computations of a Slat with a Blunt Trailing Edge,” AIAA J., Vol. 38, No. 11, pp. 2050-2058, Nov. 2000.

13. Singer, B.A., Lockard, D.P., and Brentner, K.S., "Computational Aeroacoustic Analysis of Slat Trailing-Edge Flow," AIAA J. Vol. 38, No. 9, pp. 1558-1564, Sept. 2000.

14. Khorrami, M.R., Singer, B.A., and Berkman, M.E., "Time-Accurate Simulations and Acoustic Analysis of Slat Free-Shear Layer," AIAA J., Vol. 40, No. 7, pp. 1284-1291, July 2002.

15. Khorrami, M.R., Singer, B.A., and Lockard, D.P., "Time-Accurate Simulations and Acoustic Analysis of Slat Free-Shear-Layer: Part II,” AIAA Paper 2002-2579, June 2002.

16. Choudhari, M., Khorrami, M.R., Lockard, D.P., Atkins, H., and Lilley, G., "Slat Cove Noise Modeling: A Posteriori Analysis of Unsteady RANS Simulations,” AIAA Paper 2002-2468, 2002.

17. Khorrami, M.R., Choudhari, M., Singer, B.A., Lockard, D.P., and Streett, C.L., "In Search of the Physics: The Interplay of Experiment and Computation in Slat Aeroacoustics," AIAA Paper 2003-0980, Jan. 2003.

18. Khorrami, M.R., "Understanding Slat Noise Sources," In Proceedings of EUROMECH Colloquium 449, Computational Aeroacoustics: From Acoustic Sources Modeling to Far-Field Radiated Noise Prediction, Chamonix, France, Dec. 2003.

19. Khorrami, M.R., Choudhari, M., and Jenkins, L.N, "Characterization of Unsteady Flow Structures Near Leading-Edge Slat: Part II. 2D Computations,” AIAA Paper 2004-2802, 2004. 
20. Klausmeyer, St.M. and Lin, J.C., "Comparative Results from a CFD Challenge Over a 2D Three-Element HighLift Airfoil,” NASA-TM-112858, May 1997.

21. Rumsey, C.L., and Ying, S.X., "Prediction of High Lift: Review of Present CFD Capability," Progress in Aerospace Sciences, Vol. 38, pp. 145-180, 2002.

22. Jenkins, L.N., Khorrami, M.R., and Choudhari, M., "Characterization of Unsteady Flow Structures Near Leading-Edge Slat: Part I. PIV Measurements,” AIAA Paper 2004-2801, 2004.

23. Takeda, K. Ashcroft, G.B, Zhang, X., and Nelson, P.A., "Unsteady Aerodynamics of Slat Cove Flow in a HighLift Device Configuration,” AIAA Paper 2001-0706, Jan. 2001.

24. Rumsey, C.L., LeeRausch, E.M. and Watson, R.D., "Three-Dimensional Effects in Multi-Element High Lift Computations," Computers and Fluids, Volume 32, pp. 631-657, 2003

25. Terracol, M., Labourasse, E., Manoha, E., and Sagaut, P., "Simulation of the 3D Unsteady Flow in a Slat Cove for Noise Prediction,” AIAA Paper 2003-3110, May 2003.

26. Deck, S., "Zonal-Detached-Eddy Simulation of the Flow Around a High-Lift Configuration," AIAA J., Vol. 43, No. 11, pp. 2372-2384, Nov. 2005.

27. Rumsey, C.L., Gatski, T.B., Ying, S.X and Bertelrud, A., "Prediction of High-Lift Flows Using Turbulent Closure Models," AIAA J., Vol. 36, No. 5, pp. 765-774, 1998.

28. McGinley, C.B., Anders, J.B., and Spaid, F.W., "Measurements of Reynolds Stress Profiles on a High-Lift Airfoil," AIAA Paper 1998-2620, 1998.

29. Paschal, K., Jenkins, L., and Yao, C., "Unsteady Slat-Wake Characteristics of a High-Lift Configuration,” AIAA Paper 2000-0139, Jan 2000.

30. Roger, M. and Perennes, S, "Low Frequency Noise Sources in Two-Dimensional High-Lift Devices," AIAA Paper 2000-1972, 2000.

31. Rumsey, C.L., Biedron, R.T. and Thomas, J.L. "CFL3D: Its History and Some Recent Applications," NASATM-112861, 2005.

32. Vatsa, V. and Singer, B.A., "Evaluation of a Second-Order Accurate Navier-Stokes Code for Detached Eddy Simulation Past a Circular Cylinder," AIAA Paper 2003-4085, 2003.

33. Menter, F., "Improved Two-Equation k- $\omega$ Turbulence Models for Aerodynamic Flows," NASA TM 103975, 1992.

34. Schur, M., Spalart, P.R, Squires, K.D., Strelets, M., and Travin, A., "Three-Dimensionality in Reynolds Averaged Navier-Stokes Solutions Around Two-Dimensional Geometries," AIAA J., Vol. 43, No. 6, pp. 1230-1242, 2005.

35. Chandrsuda C., Mehta, R.D., Weir, A.D., and Bradshaw, P., "Effect of Free-Stream Turbulence on Large Structure in Turbulent Mixing Layers," J. Fluid Mech., Vol. 85, pp. 693-704, 1978.

36. Browand, F.K. and Troutt, T.R., "A Note on Spanwise Structure in the Two-Dimensional Mixing Layer," J. Fluid Mech., Vol. 91, pt. 4, pp. 771-781, 1980.

36. Bernal, L.P. and Roshko, A., "Streamwise Vortex Structures in Plane Mixing Layers," Vol. 170, pp. 499-525, 1986. 
38. Bell, J.H. and Mehta, R.D., "Measurements of the Streamwise Vortical Structures in a Plane Mixing Layer," J. Fluid Mech., Vol. 239, pp. 213-248, 1992.

39. Comte, P., Lesieur, M. and Lamballais, E., "Large- and small-scale stirring of vorticity and a passive scalar in a 3-D temporal mixing layer,” Phys. Fluids A, Vol. 4, No. 12, pp. 2761-2778, 1992.

40. Collis, S.C., Lele, S.K., Moser, R.D. and Rogers, M.M. "The Evolution of Plane Mixing Layer with Spanwise Non-uniform Mixing,” Phys. Fluids, Vol. 6, No. 1, pp. 381-396, 1994.

41. Kendall, J.M., "Experiments on Boundary-Layer Receptivity to Freestream Turbulence," AIAA Paper 19980530, 1998.

42. Olson, S., Thomas, F.O., and Nelson, R. C., "Mechanisms of Slat Noise Production in a 2D Multi-Element Airfoil Configuration.," AIAA Paper 2001-2156, 2001.

43. Takeda, K., Ashcroft, G.B., Zhang, X. and Nelson, P.A., "Unsteady Aerodynamics of Flap Cove Flow in a HighLift Device Configuration," AIAA Paper 2001-0707, Jan. 2001.

44. Jimenez, J., Martinez-Val, R., and Rebollo, M., "On the Origin and Evolution of Three-Dimensional Effects in the Mixing Layer," Univ. of Madrid Final Report on U.S. Army Grant DA-ERO 78-G-079, Dec. 1979.

45. Bell, J.H., Plesniak, M.W. and Mehta, R.D., "Spanwise Averaging of Plane Mixing Layer Properties," AIAA J., Vol. 30, No. 3, pp. 835-837, 1992.

46. Hill, W.G., Jr., Jenkins, R.C., and Gilbert, B.L., "Effects of the Initial Boundary-Layer State on Turbulent Jet Mixing," AIAA J., Vol. 14, No. 11, pp. 1513-1514, 1976.

47. Ho, C.-M. and Huerre, P., "Perturbed Free Shear Layers,” Ann. Rev. Fluid Mech., Vol. 16, pp. 365-424, 1984.

48. Moriarty, P. and Heineck, J.T., PIV Measurements Near a Leading Edge Slat," Proc. of the $3^{\text {rd }}$ Intl. Workshop on PIV, 1999.

49. Brooks, T.F., and Humphreys, T., "Flap Edge Aeroacoustic Measurements and Predictions," AIAA Paper 2000$1975,2000$.

50. Guo, Y.-P., Joshi, M.C., Bent, P.H., and Yamamoto, K.J., "Surface Pressure Fluctuations on Aircraft Flaps and Their Correlation with Far-Field Noise,” J. Fluid Mech.,Vol. 415, pp. 175-202, 2000.

51. Guo, Y.-P., “A Discrete Vortex Model for Slat Noise Prediction,” AIAA Paper 2001-2157, 2001.

52. Mabey, F.G., "Analysis and Correlation of Data on Pressure Fluctuations in Separated Flow," J. Aircraft, Vol. 9 , No. 9, pp. 642-645, 1972.

53. Takeda, K. Zhang, X., and Nelson, P.A., "Unsteady Aerodynamics and Aeroacoustics of a High-Lift Device Configuration," AIAA Paper 2002-0570, Jan. 2002.

54. Jones, B. G., Planchon, H. P., and Hammersley, R. J., "Turbulent correlation measurements in a two-stream mixing layer.," AIAA J., Vol.11 No.8, pp. 1146-1150. 1973.

55. Zheng, T. and Povitsky, A., "Sound Generation by Street of Vortices in a Non-Uniform Flow", AIAA Paper 2005-5160, 2005.

56. Agarwal, A. and Morris, P., "Broadband Noise from the Unsteady Flow in a Slat Cove," AIAA Paper 20040854, 2004. 


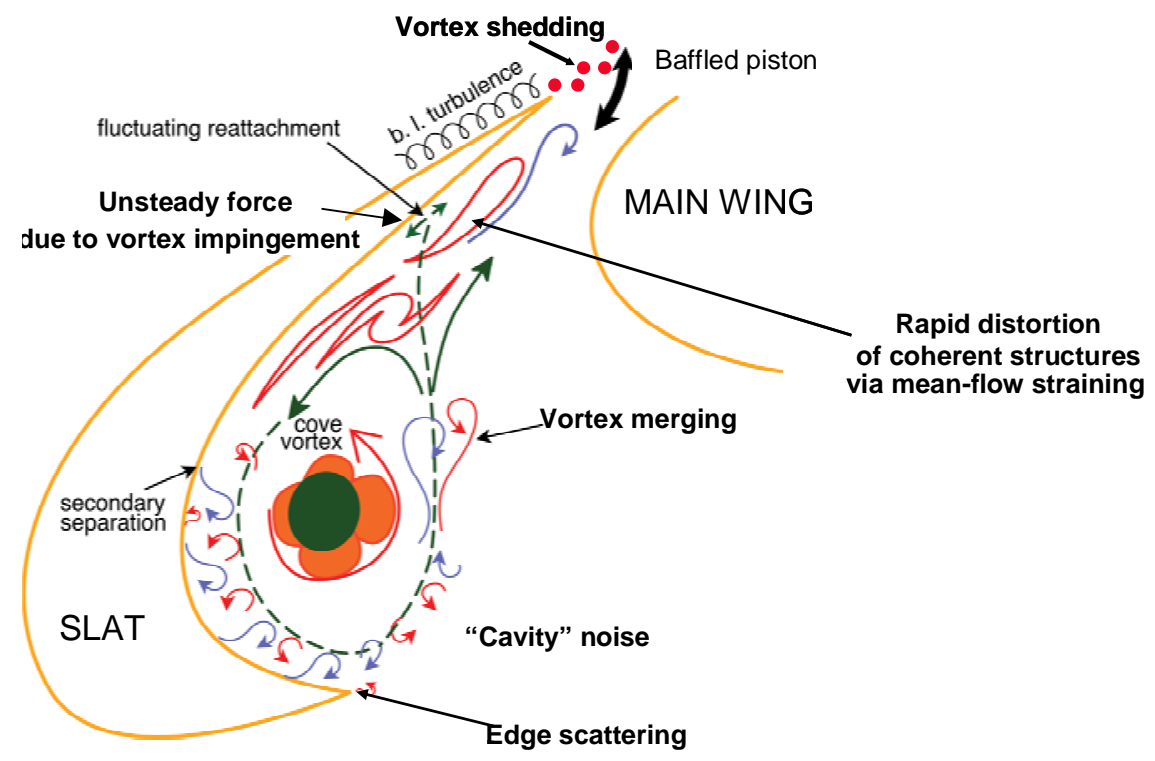

Figure 1: Potential sources and physical mechanisms behind noise generation near a leading edge slat

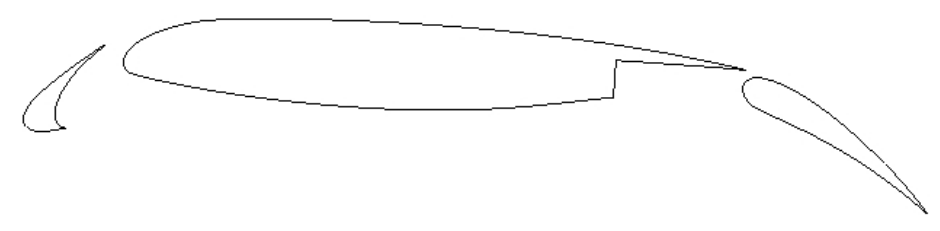

Fig. 2. Cross-sectional view of three-element 30P30N high-lift system.

slat

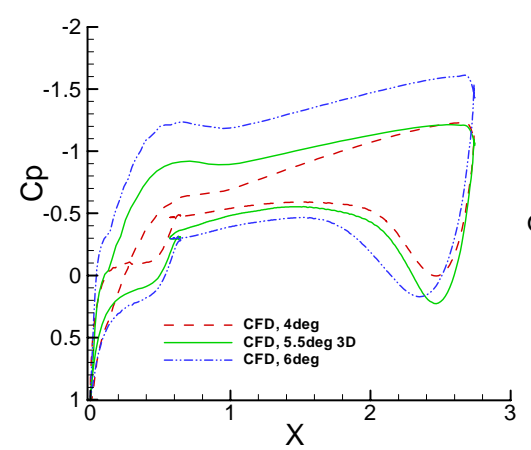

Main element

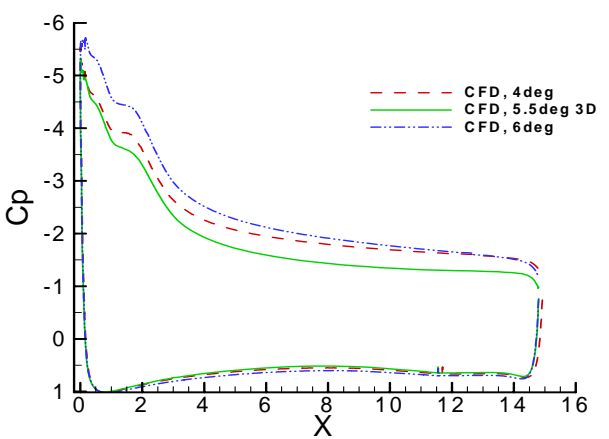

flap

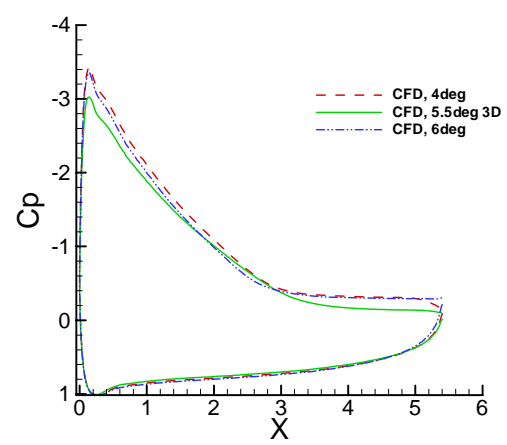

Figure 3: Comparison between mean surface pressure distribution for free-flight configuration (5.5 deg AOA) and 2D RANS CFD for tunnel installed model at 4 and 6 degree AOA [19]. The latter predictions have been shown to agree with static pressure measurements in BART facility at NASA Langley Research Center [22]. Abscissa in each of the above plots denotes the streamwise coordinate (in inches) relative to the leading edge of the respective element. 


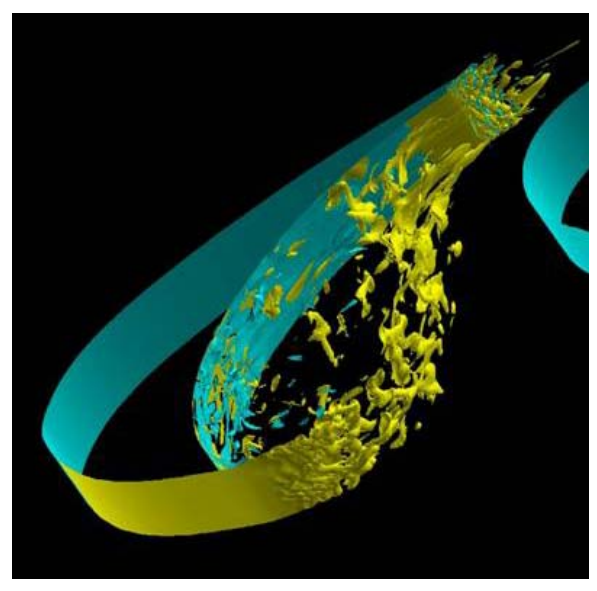

a) Spanwise vorticity field (yellow contours: $\omega_{z} \mathrm{c} / \mathrm{U}_{\infty}=265$; cyan contours: $\omega_{\mathrm{z}} \mathrm{c} / \mathrm{U}_{\infty}=-265$ )
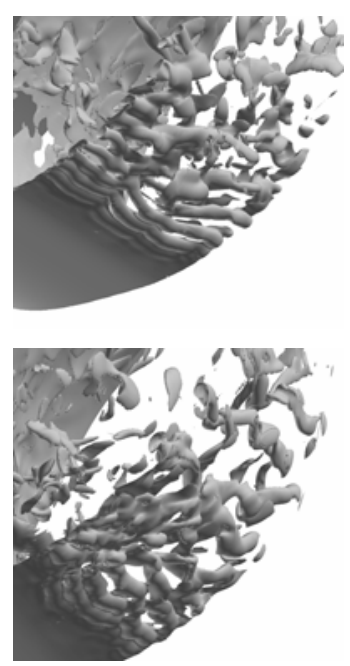

b) Vorticity magnitude near cusp

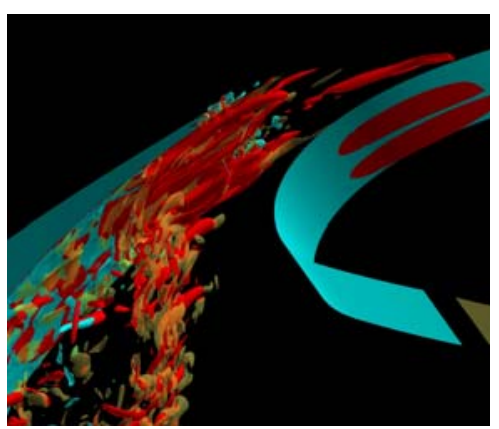

c) Spanwise vorticity (yellow and cyan) and magnitude of xy-plane vorticity (red)

Figure 4: Sample isosurfaces of instantaneous vorticity field from grid S2 simulation

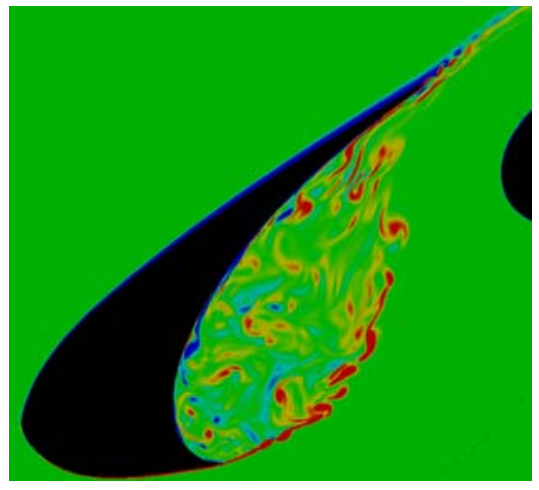

a) Planar cut resembling cove state I

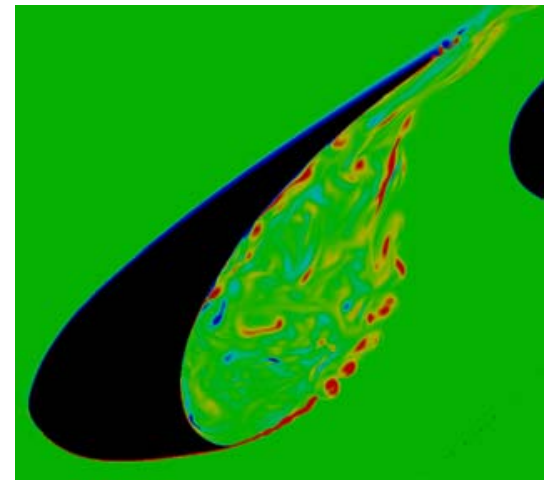

b) Planar cut somewhat resembling cove state II

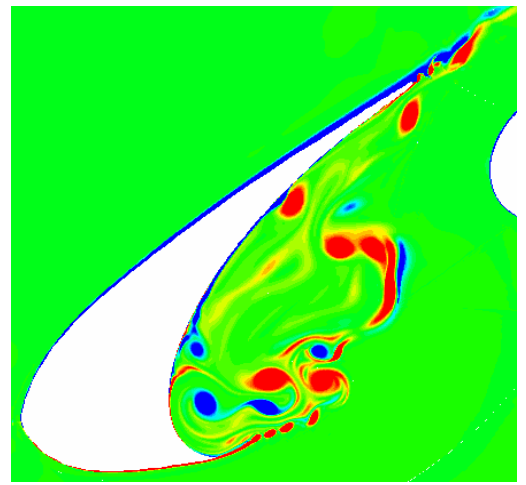

Figure 6: Instantaneous vorticity field based on 2D computations.

Figure 5: Instantaneous vorticity field based on 3D computations

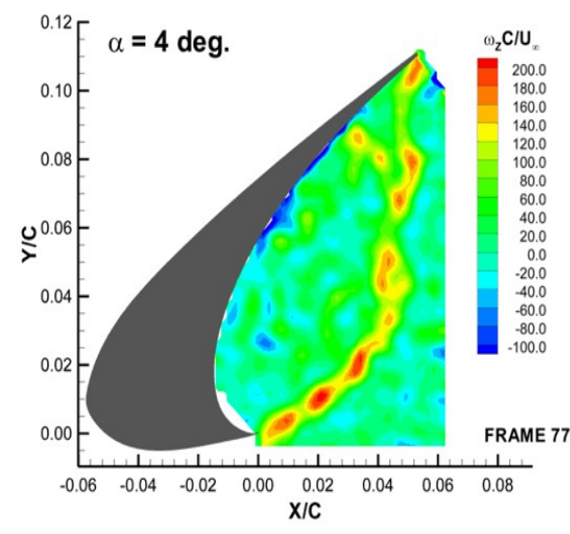

a) Cove state I

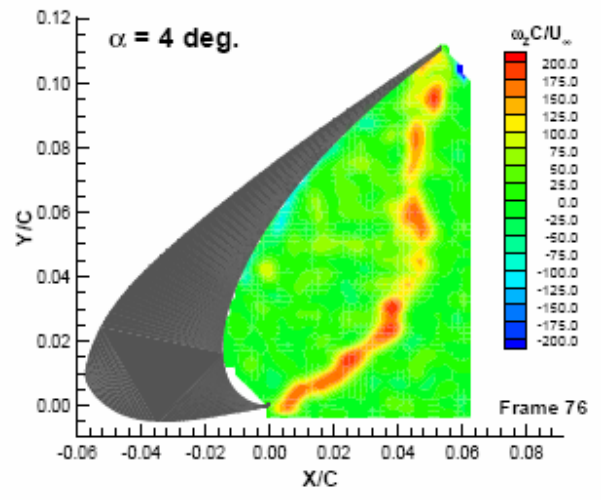

b) Cove state II

Figure 7: Sample snapshots of instantaneous, spanwise vorticity field from PIV measurements ${ }^{22}$ 

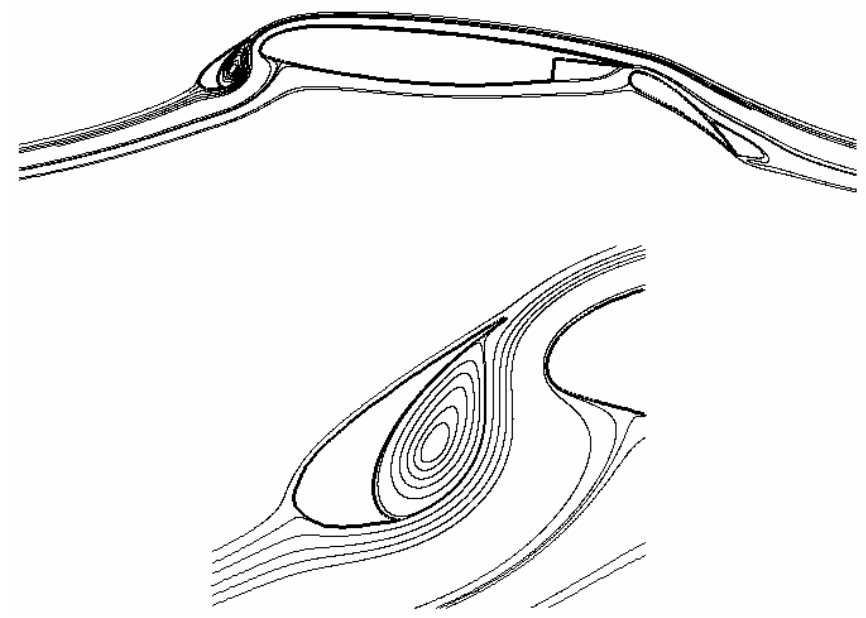

Figure 8: Mean flow streamlines based on grid S1 simulation (mean flow averaged in both time and spanwise coordinate)

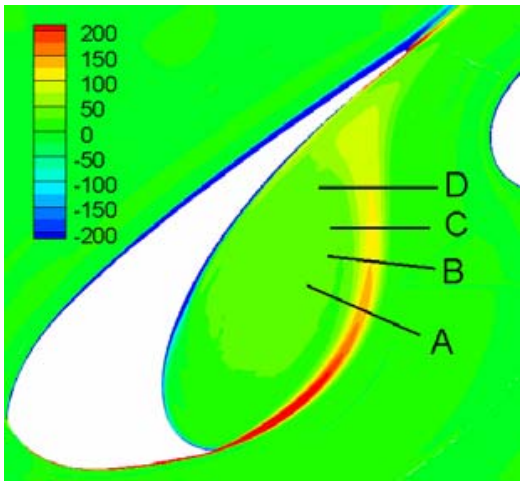

a) Locations of mixing layer profiles A through D superposed on contours of mean spanwise vorticity $\omega_{z} \mathrm{c} / \mathrm{U}_{\infty}$

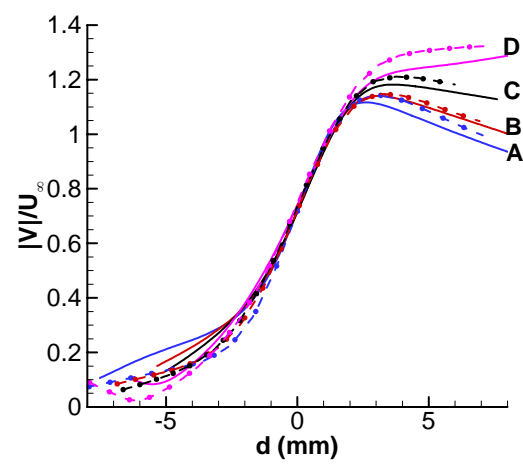

b) Results of grid S1 simuation (solid lines) compared with PIV data (broken lines)

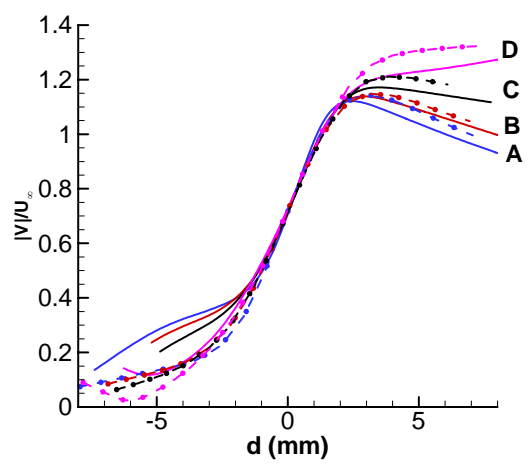

c) Results of grid S2 simulation (solid lines) compared with PIV data (broken lines)

Figure 9: Time averaged velocity magnitude profiles across slat shear layer 

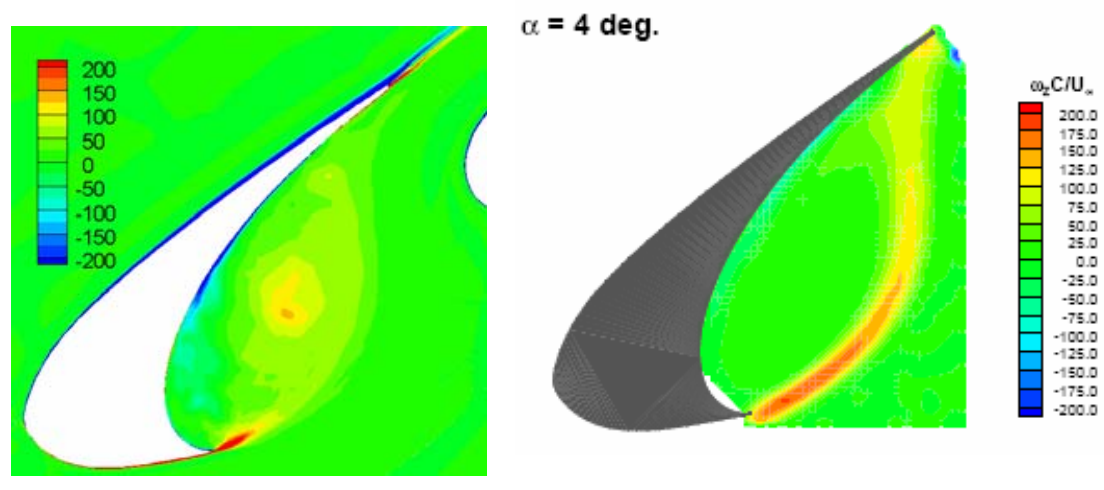

a) 2D CFD at large times

b) PIV data from Ref. [22]

Figure 10: Time averaged spanwise vorticity field $\omega_{z} \mathrm{c} / \mathrm{U}_{\infty}$ in slat cove region

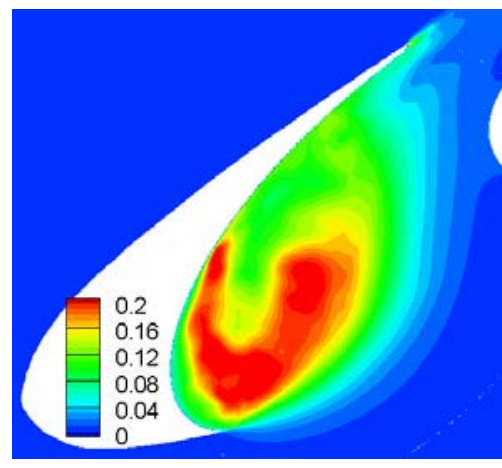

a) 2D CFD at large times

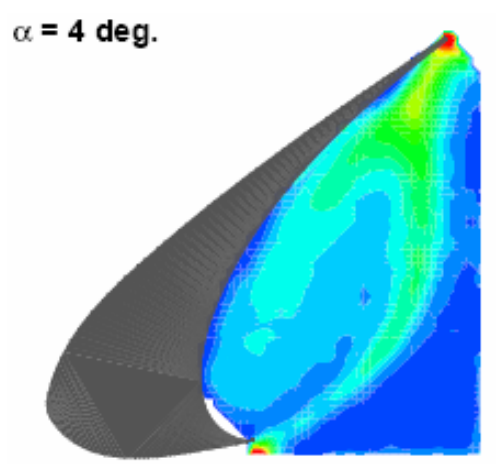

b) PIV data from Ref. [22]

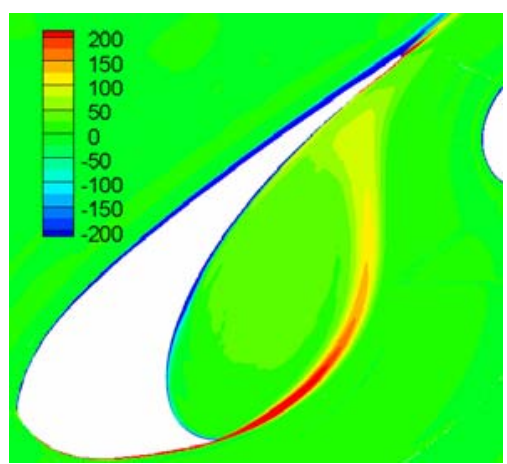

c) Present $3 \mathrm{D}$ simulations

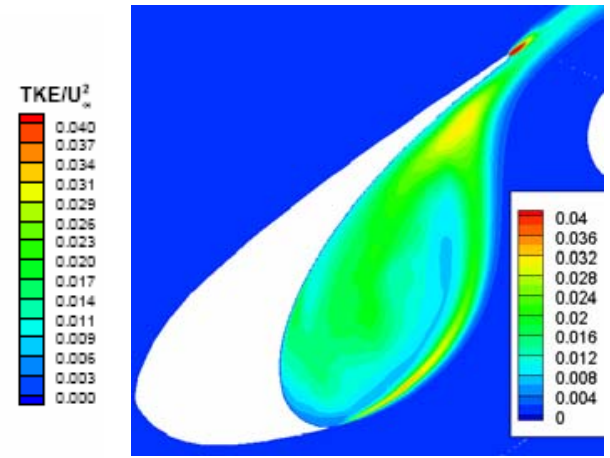

c) Present 3D simulations

Figure 11: Time averaged, 2D TKE distribution in slat cove region

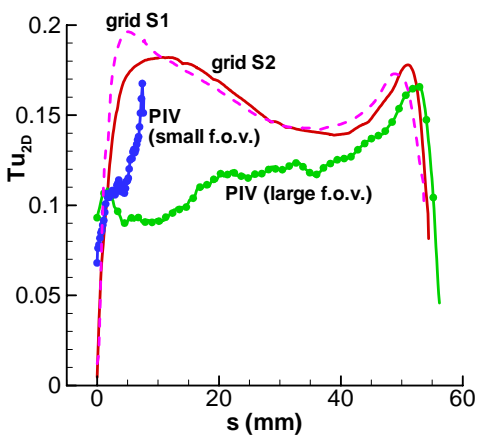

a) 2D turbulence intensity (based on u'-v' fluctuations); PIV data provided by Jenkins [22]

Figure 12: Peak turbulent fluctuations along mixing layer trajectory (s denotes distance from cusp)

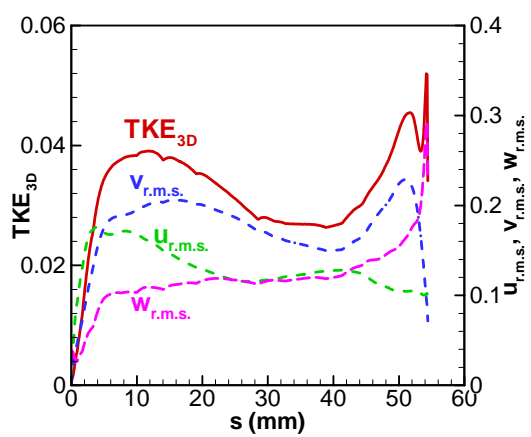

b) 3D TKE and r.m.s. velocity fluctuations

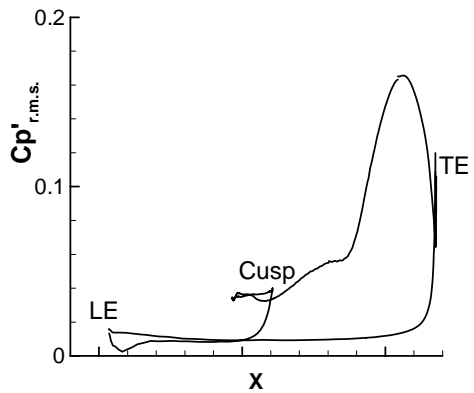

Figure 13: Root mean square pressure fluctuations along slat surface 


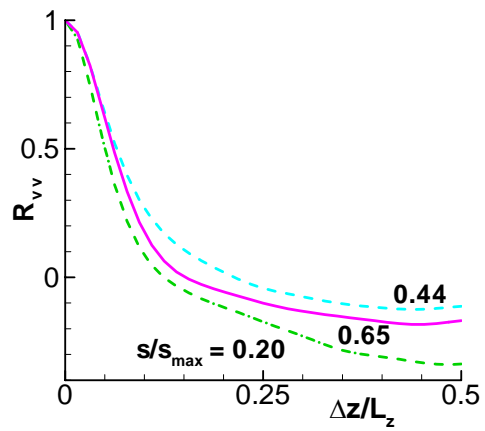

a) Correlation $R_{v v}$ in mixing layer (grid S2 simulation)

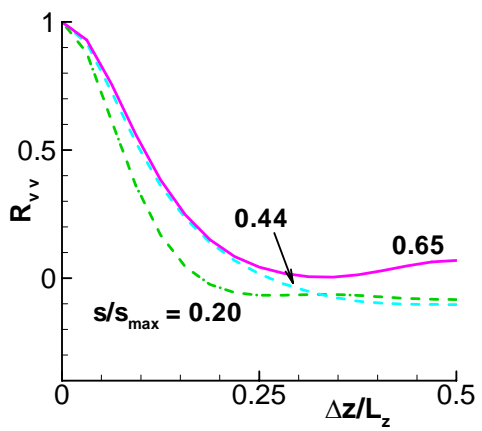

b) Correlation $R_{v v}$ in mixing layer (grid S1 simulation)

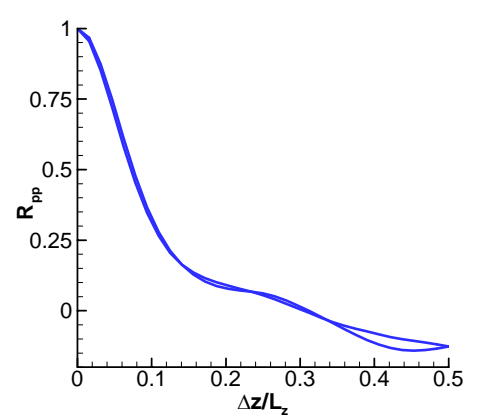

c) Correlation $R_{p p}$ near location of peak p' along slat lower surface The two curves denote positive and negative $\Delta z$, respectively (grid S2 simulation)

Figure 14: Spanwise correlations of instantaneous fluctuations in slat cove region

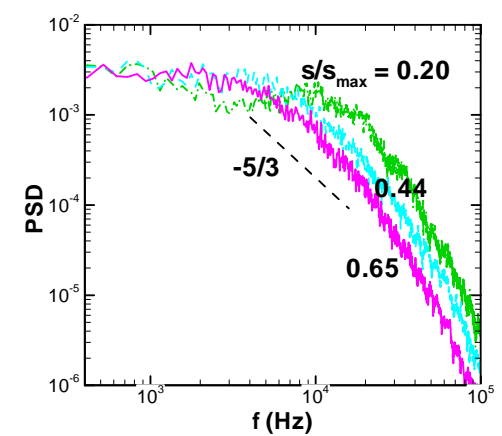

a) v' fluctuations within slat shear layer

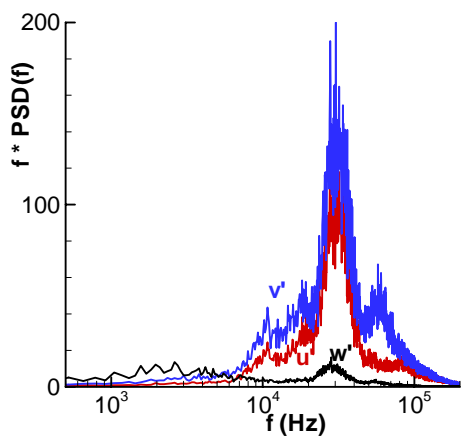

b) Velocity fluctuations in slat near wake

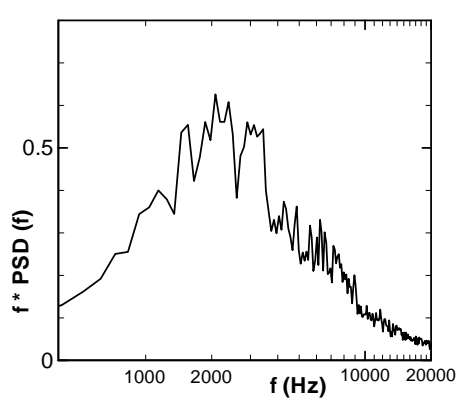

c) p' fluctuations near reattachment location

Figure 15: Frequency spectra of fluctuations near slat

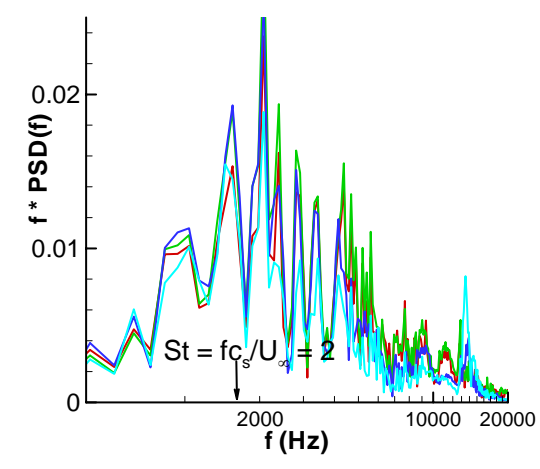

a) Frequency spectra of computed acoustic pressure at selected probe locations, at distances of between 1-2 $\mathrm{c}_{\mathrm{s}}$ from the slat trailing edge

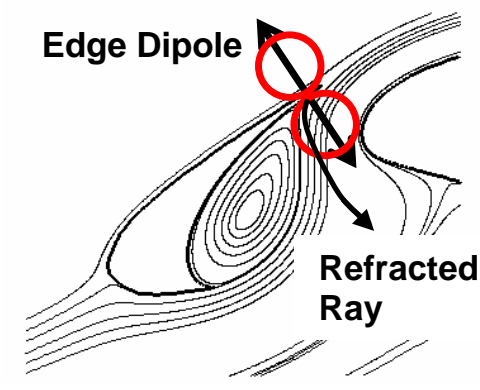

b) Schematic of slat noise model proposed by Dobrzynski and PottPollenske (based on Ref. [8])

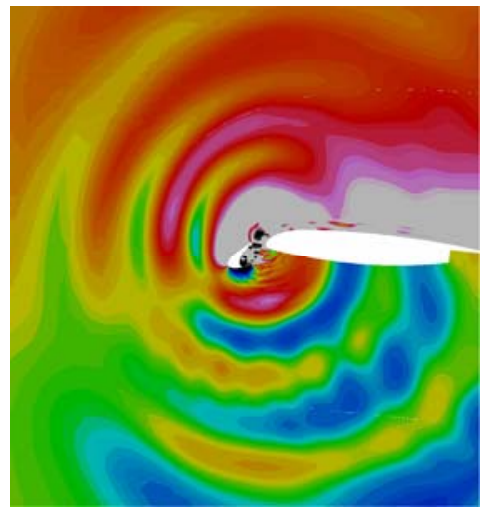

c) Contours of instantaneous pressure perturbations based on grid S2 simulation

Figure 16: Acoustic radiation due to slat cove unsteadiness 